\title{
Differenzialdiagnosen von Erosionen
}

Nadine Schlüter, Carolina Ganß

\section{Zur Klasse der nicht kariesbedingten Zahnhartsubstanzdefekte oder nicht kariösen Verschleißformen gehören Erosionen, Abrasionen - inklusive der Sonderform Demastikation -, Attritionen sowie der keilförmige Defekt. Während Erosionen durch den Einfluss von Säuren, also chemisch bedingt sind, sind die anderen Formen mechanisch induziert.}

Die Therapie bei nicht kariesbedingten Zahnhartsubstanzverlusten ist primär kausal ausgerichtet [1], da der Verschleiß nicht weiter voranschreitet, wenn die verursachende Noxe nicht mehr einwirkt. Es ist daher besonders wichtig, die Ursache für klinisch diagnostizierte Defekte sicher identifizieren zu können. Neben einer Anamnese kommt der Beurteilung der Defektmorphologie eine zentrale Bedeutung zu, um die verschiedenen Verschleißformen sicher voneinander abgrenzen und somit wirksame und nachhaltige Therapiestrategien entwickeln zu können. Allerdings können mechanische und chemische Noxen zeitgleich in der Mundhöhle auftreten. So ist beispielsweise ein Zusammenspiel von Erosionen und Abrasionen keine Seltenheit, was zumeist als erosiv induzierter Zahnverschleiß bezeichnet wird (aus dem Englischen: erosive tooth wear). Das kann einerseits zu einer gegenseitigen Verstärkung und damit Erhöhung der Progression der Substanzverluste führen [2]. Auf der anderen Seite wird dadurch ebenfalls das Erscheinungsbild der Defekte in der Mundhöhle modifiziert, was eine klare Zuordnung zu einem Ursachenkomplex erschweren kann [3].

Traditionsgemäß werden die Defekte nach ihrer Ursache oder Morphologie benannt. Der Begriff Attrition leitet sich aus dem Lateinischen „atterere“ ab, was so viel bedeutet wie „reiben, scheuern“. Bei Attritionen handelt es sich um einen klassischen 2-Medien-Verschleiß; 2 Substanzen, in diesem Fall die Zahnhartsubstanz antagonistischer Zähne, reiben aufeinander. Das klassische Erscheinungsbild einer Attrition sind scharf begrenzte, plane und hochglänzende Facetten auf den Okklusalflächen ( $\bullet$ Abb. 1), wobei kongruente Flächen an Antagonisten gefunden werden ( $\bullet$ Abb. 2). Sehr ausgeprägte Defekte können zu einem Verlust der Kronenhöhe führen. Attritionen finden sich immer im okklusionsgetragenen Bereich ( $\bullet$ Abb. 8 a), Attritionsdefekte auf den Glattflächen gibt es nicht.

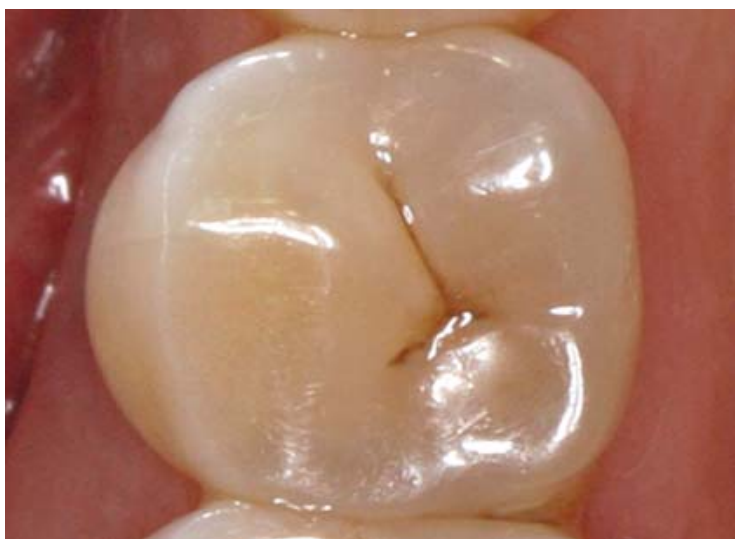

- Abb. 1 Attritionsdefekt auf dem bukkalen Höcker eines UK-Prämolaren. Die hochglänzende Facette ist deutlich zu erkennen.

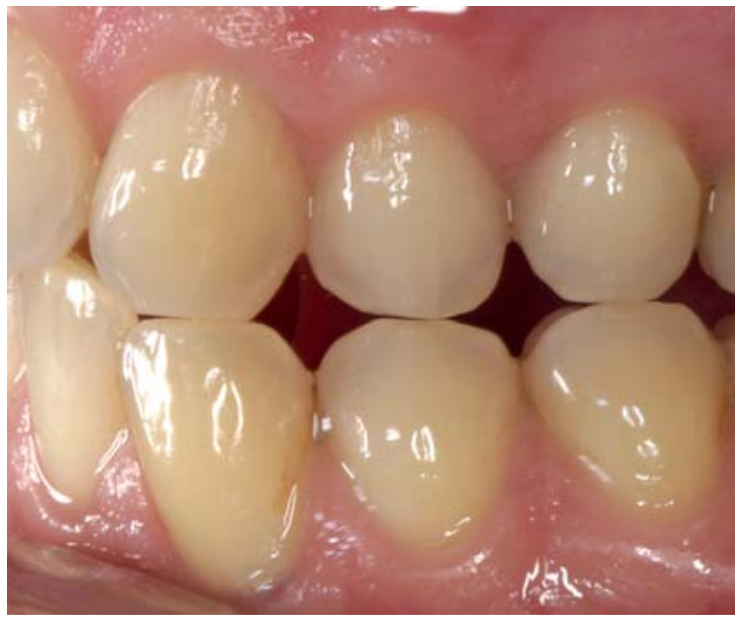

- Abb. 2 Kongruente Flächen an Antagonisten bei Attritionen. 


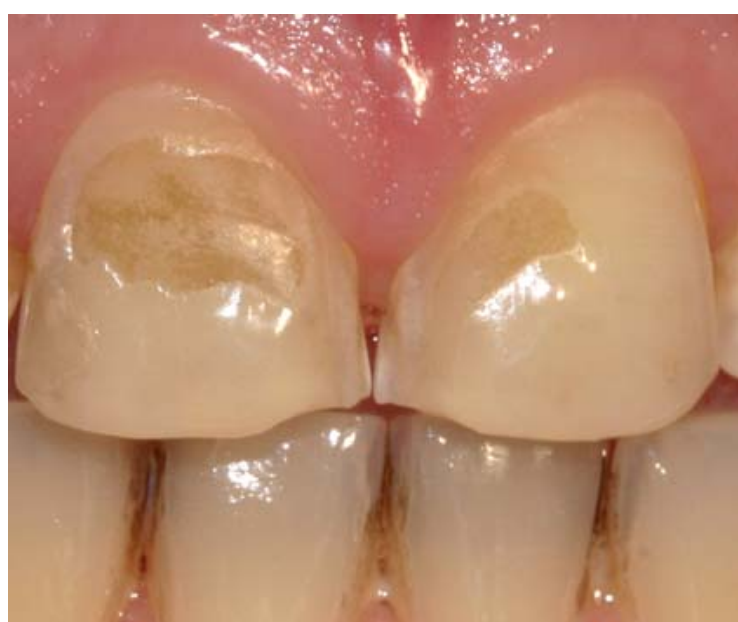

- Abb. 3 Erosionen an den Vestibulärflächen von Oberkiefer-Inzisivi. Das Dentin ist durch sekundäre Farbstoffeinlagerung dunkel verfärbt. Der Patient hat regelmäßig beim Sport Getränke aus einer Sportlerflasche zu sich genommen. Die Flasche hat er mit den Zähnen geöffnet, was zusätzlich zu den Erosionen zu Abrasionen an der Inzisalkante geführt hat.

Abrasionen sind ein Vertreter des 3-Medien-Verschleißes. Dabei können die 3 Medien sehr unterschiedlich sein. So können Zahnpaste und Zahnbürste, aber auch Gewohn-

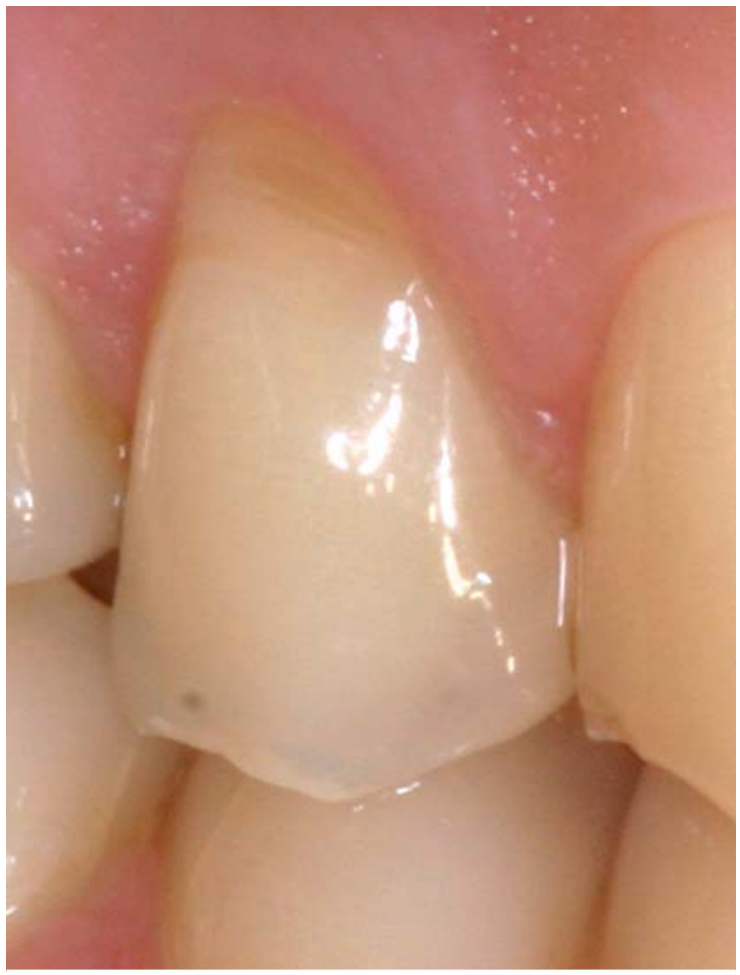

- Abb. 4 Bürstabrasionen aufgrund einer horizontalen Bürsttechnik mit zu viel Druck. Deutlich zu erkennen sind die durch die Zahnbürste verursachten Rillen im Defektbereich. heiten, wie das Halten von Gegenständen mit den Zähnen oder das Benutzen von Zähnen als Werkzeug ( $\bullet$ Abb.3), eine Rolle spielen. Werden die Abrasionen durch Schleifpartikel in der Nahrung hervorgerufen, werden sie Demastikation genannt. Beide Begriffe leiten sich ebenfalls aus dem Lateinischen ab; Abrasion kommt von „abradere: abkratzen, abscheren“ und Demastikation von „mandere: kauen, beißen, essen, verzehren“. Die Morphologie von Abrasionsdefekten kann sehr vielfältig sein und entspricht der einwirkenden abrasiven Noxe. Typisch für Defekte aufgrund von Bürstabrasion sind amorphe Defekte zumeist unterhalb der Schmelz-Zement-Grenze auf freigelegten Wurzeloberflächen ( $\bullet$ Abb. 4). Demastikationen lassen sich ausschließlich okklusal diagnostizieren. Es liegen in der Regel muldenförmige Defekte vor. Sie haben eine ähnliche Form wie erosiv bedingte okklusale Defekte.

Der keilförmige Defekt wird vermutlich durch eine Kombination aus Abfraktion und Abrasion verursacht. Abfraktionen, vom Lateinischen „frangere: brechen“, können durch eine okklusale Überbelastung entstehen. Durch Belastungsspitzen kommt es im zervikalen Bereich zu Spannungen, sodass kleine Zahnhartsubstanzpartikel aus diesem Bereich herausgesprengt werden. Vermutlich dienen diese sehr initialen Defekte später beispielsweise bei einer Mundhygiene mit horizontalem Putzmuster der

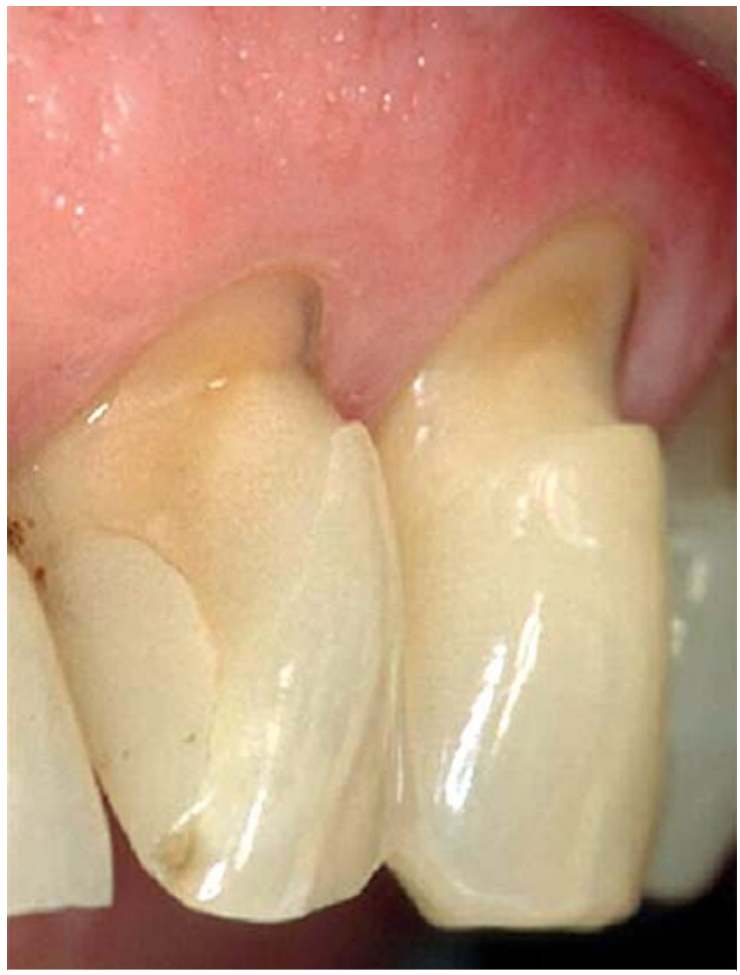

- Abb. 5 Typische keilförmige Defekte mit Ursprung an der Schmelz-Zement-Grenze (die Grenze liegt hier senkrecht zur Zahnoberflächen) und einem Substanzverlust, der zervikal flach ausläuft. 


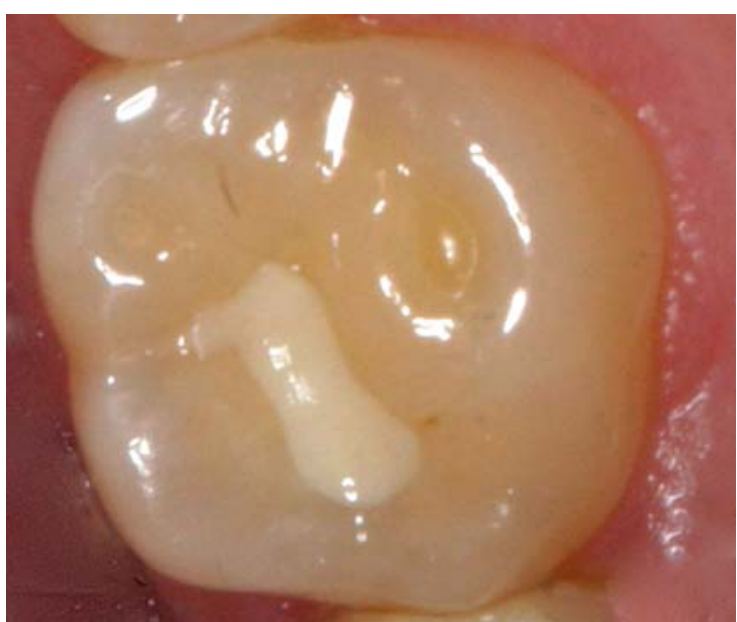

- Abb. 6 Typischer muldenförmiger Erosionsdefekt auf den Höckerspitzen eines Oberkiefer-Molaren.
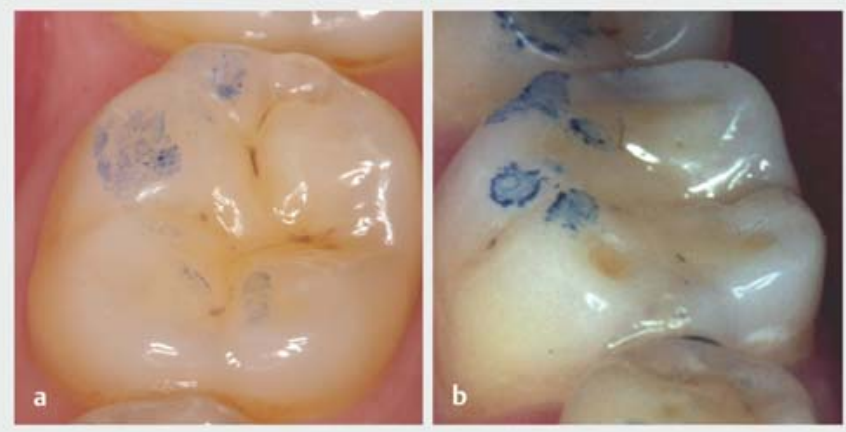

- Abb. 8 Darstellung des Okklusionsmusters bei Attritionen (a) und Erosionen (b). Bei Attritionen befinden sich die Defekte immer im Kontaktbereich, bei Erosionen außerhalb.

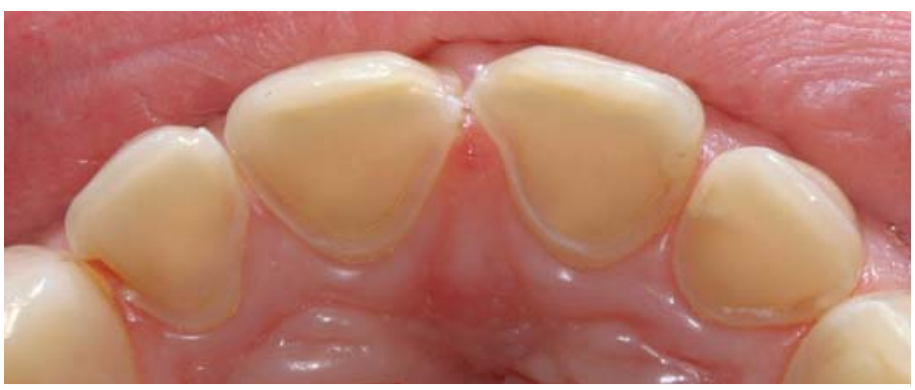

- Abb. 9 Ausgeprägte Erosionen palatinal an den Oberkiefer-Inzisivi. Trotz der starken Ausprägung der Defekte ist zervikal ein intaktes Schmelzband zu erkennen.

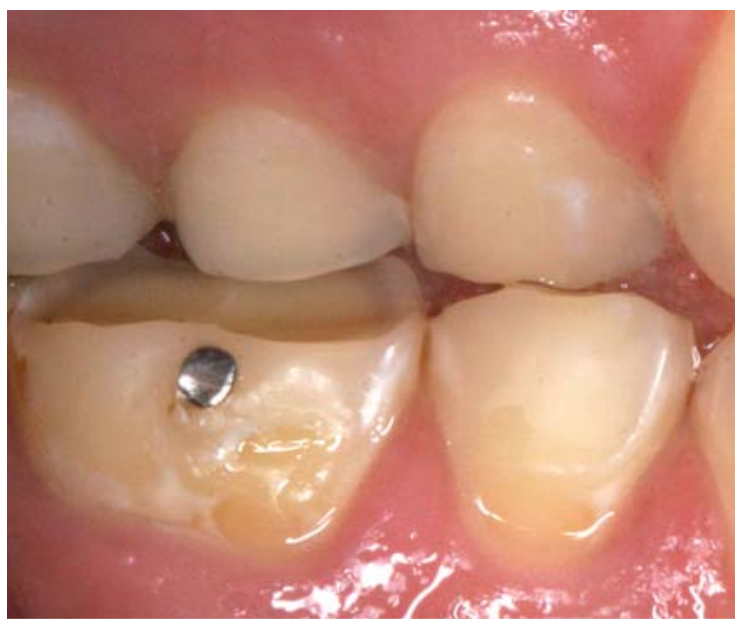

- Abb. 7 Patient mit ausgeprägten Erosionen. Es ist deutlich ein Verlust der Kronenhöhe zu erkennen.

Zahnbürste als Führungsrille, was zu einer Verstärkung der Substanzverluste führen kann. Ob dieser Mechanismus tatsächlich eine Rolle spielt, ist nicht vollständig geklärt, allerdings spricht das mitunter im Defektbereich anzutreffende horizontale Rillenmuster für eine derartige Beteiligung der Zahnbürste. Keilförmige Defekte sind ausschließlich auf den Glattflächen zu finden, im Regelfall vestibulär. Sie nehmen ihren Ursprung an der SchmelzZement-Grenze - die Grenze liegt hier senkrecht zur Zahnoberflächen - und laufen nach zervikal hin oft flach aus ( $\sim$ Abb. 5). Die Tiefe der Defekte übersteigt in der Regel deren Breitenausdehnung [4,5].

Die chemisch bedingten Erosionen (lateinisch „rodere: nagen“) können sich sowohl auf den Okklusal- als auch auf den Glattflächen manifestieren. Typisches Zeichen okklusaler Erosionen sind muldenförmige Defekte auf den Höckerspitzen ( $\bullet \mathbf{A b b}$. 6) und eine Einebnung des Höcker-Fissuren-Reliefs mit Verlust der Kronenhöhe bei ausgeprägten Defekten ( $\bullet$ Abb. 7). Im Gegensatz zu Attritionen ( $\bullet \mathbf{A b b}$. 8a) liegen Erosionen nicht im kontaktpunktgetragenen Bereich ( $\bullet \mathbf{A b b} \mathbf{8} \mathbf{8} \mathbf{b}$ ). Auf den Glattflächen beginnen Erosionen mit einer initialen Verarmung an typischen Oberflächenstrukturen, wie Glanz und Perikymatien. Später folgen eine Abflachung der konvexen Areale sowie anschließend muldenförmige Defekte, die zervikal von einem intakten Schmelzband begrenzt sind ( $\bullet$ Abb. 9). Die Breite der Defekte übersteigt in der Regel deren Tiefenausdehnung, sodass sie dadurch gut von keilförmigen Defekten zu unterscheiden sind. Glattflächenerosionen können sowohl palatinal als auch vestibulär auftreten; linguale Erosionen sind sehr selten [6]. 
Interessenkonflikt

Frau Prof. Dr. Nadine Schlüter erklärt, dass sie für die Fa. Colgate und die Fa. EMS in den letzten drei Jahren Vortragstätigkeiten ausgeübt hat. Frau Prof. Dr. Carolina Ganß erklärt, dass sie in den letzten drei Jahren durch die Fa. Colgate finanzierte Vortragstätigkeiten ausgeübt hat. Weitere wirtschaftliche oder persönliche Verbindungen zwischen den Autoren und Organisationen im Sinne eines Interessenskonflikts bestehen und bestanden nicht.

Autorinnen/Autoren

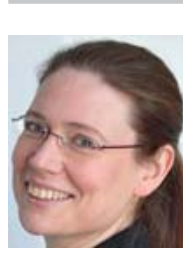

\section{Prof. Dr. Nadine Schlüter}

Stiftungsprofessur für Kariesforschung, Klinik für Zahnerhaltungskunde und Parodontologie, Department f. Zahn-, Mund- u. Kieferheilkunde, Universitätsklinikum Freiburg, Medizinische Fakultät, Albert-LudwigsUniversität Freiburg, Deutschland

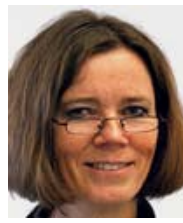

\section{Prof. Dr. Carolina Ganß}

Oberärztin an der Poliklinik für Zahnerhaltungskunde und Präventive Zahnheilkunde, Medizinisches Zentrum für Zahn-, Mund- und Kieferheilkunde, Justus-Liebig-Universität, Gießen

Korrespondenzadresse

Prof. Dr. Nadine Schlüter

Stiftungsprofessur für Kariesforschung

Klinik für Zahnerhaltungskunde und Parodontologie

Department f. Zahn-, Mund- u. Kieferheilkunde

Universitätsklinikum Freiburg

Hugstetter Straße 55

79106 Freiburg i. Br.

nadine.schlueter@uniklinik-freiburg.de

\section{Literatur}

[1] Lussi A, Hellwig E. Risk assessment and causal preventive measures. Monogr Oral Sci 2014; 25: 220-229

[2] Wiegand A, Schlueter $N$. The role of oral hygiene: does toothbrushing harm? Monogr Oral Sci 2014; 25: 215-219

[3] Shellis RP, Addy M. The interactions between attrition, abrasion and erosion in tooth wear. Monogr Oral Sci 2014; 25 : 32-45. doi:10.1159/000359936

[4] Ganss C, Schlueter N. Diagnosis of dental Erosion. In: Amaechi BT, ed. Dental Erosion and its clinical Management. Cham, Heidelberg, New York, Dordrecht, London: Springer; 2015: 99-110

[5] Ganss C, Lussi A. Diagnosis of erosive tooth wear. Monogr Oral Sci 2014; 25: 22-31. doi:10.1159/000359935

[6] Ganss C, Schlueter N. Diagnosis of dental erosion. Clin Dent Rev 2017; 1: 12. doi:10.1007/s41894-017-0012-2

Bibliografie

DOI https://doi.org/10.1055/a-0715-3570

ZWR - Das Deutsche Zahnärzteblatt 2018; 127: 502-505

(c) Georg Thieme Verlag KG Stuttgart · New York

ISSN 0044-166X 